\title{
LES-DFSD Modelling of Turbulent Premixed Flames Past Repeated Obstacles
}

\author{
Ruipengyu $\mathrm{Li}^{1}$, Weeratunge Malalasekera ${ }^{1}$, Salah Ibrahim ${ }^{2}$ \\ ${ }^{1}$ Wolfson School of Mechanical, Electrical and Manufacturing Engineering, Loughborough University \\ Loughborough, LE11 3TU, UK \\ r.li3@lboro.ac.uk; w.malalasekera@lboro.ac.uk \\ ${ }^{2}$ Department of Aeronautical and Automotive Engineering, Loughborough University \\ Loughborough, LE11 3TU, UK \\ s.s.ibrahim@lboro.ac.uk
}

\begin{abstract}
This paper presents simulations of propagating turbulent premixed deflagrating flames past built-in solid obstructions in a small-scale combustion chamber. The design of the chamber allows for up to three baffle plates and a central square obstacle to be positioned in the path of the propagating flames in order to generate turbulence and increase the flame propagating speed. The test case considered in this paper uses a stagnant, stoichiometric propane-air mixture in the configuration of three baffles and one central obstacle. Simulations have been carried out with the Large Eddy Simulation (LES) technique. The filtered reaction rate in LES is accounted for using a novel dynamic flame surface density (DFSD) model. Both numerical and experimental results show that the flame is initially laminar and becomes fully turbulent after continuous interaction with the obstacles downstream. Satisfactory agreement made between the LES calculations and the experimental data confirms the capability of the DFSD model in reproducing essential flame characteristic parameters including the maximum overpressure and flame front speed. The interaction between obstaclegenerated turbulence and the flame front is quantified using the sub-grid-scale (SGS) wrinkling factor. Various stages of flame propagation and the dynamic behaviours of the flame are also examined based on the evolution and spatial distribution of the wrinkling factor.
\end{abstract}

Keywords: Turbulent Premixed Flames; LES; Dynamic Flame Surface Density Model; Repeated Obstacles.

$\begin{array}{ll}\text { Nomenclature } \\ \rho: & \text { Density } \\ \Xi_{\Delta}: & \text { Sub-grid-scale wrinkling factor } \\ c: & \text { Reaction progress variable } \\ u_{i}: & \text { Velocity component in } i(\mathrm{x}, \mathrm{y} \text { and } \mathrm{z}) \text { direction } \\ \rho_{u}: & \text { Density of the unburned mixture } \\ s_{L}^{0}: & \text { Unstrained laminar burning velocity } \\ \delta_{L}^{0}: & \text { Unstrained laminar flame thickness } \\ \delta_{c}: & \text { Inner cut-off scale } \\ \Delta: & \text { Filter size } \\ \hat{\Delta}: & \text { Test-filter size } \\ \beta: & \text { Model parameter for the dynamic model } \\ \langle\cdots\rangle: & \text { Volume averaging } \\ \hat{\cdots}: & \text { Test filtering process }\end{array}$

\section{Introduction}

Turbulent premixed flames are encountered in many engineering applications such as spark ignition engines, gas turbines and accidental explosions. Modelling of unsteady turbulent premixed propagating flames remains a challenging problem, particularly with respect to the closure of the chemical source term and adequate representation of the reaction 
zone. A deflagration is often highly transient, and the flame can accelerate significantly while interacting with surroundings of complex shapes. The generated high overpressure is responsible for the major destruction involved in an explosion event. Thus, the ability to predict the overpressure, rate of pressure rise and flame propagation speed in such scenarios is important for safe design of buildings and process plants.

The Large Eddy Simulation (LES) technique is now accepted as a feasible computational tool for detailed study of complex combustion processes. The crucial issue of using LES for premixed combustion modelling lies in the development of adequate sub-grid-scale (SGS) models that are capable of representing combustion over a wide range of conditions. As the flame is generally thinner than the typical mesh size in LES, computing combustion requires appropriate sub-models to account for the filtered chemical source term. There have been several methods proposed for the closure of the reaction rate and the flame surface density (FSD) approach based on the flamelet concept has been considered promising in calculating unsteady deflagrating flames [1,2]. The algebraic FSD model often relates the flame surface density $\Sigma$ as a function of a reaction progress variable [3] with a model parameter representing the level of flame-front wrinkling at SGS. However, an apparent disadvantage of the algebraic formulation of FSD is that the model parameter [3] usually requires a certain degree of tuning to match the experimental measurements. A possible improvement is to dynamically calculate the model parameter [4] based on the local flow and flame conditions. Such combustion model is attractive because the flame can transit from early laminar to fully turbulent in a deflagration interacting with repeated obstructions.

In the present work, a dynamic flame surface density (DFSD) model [4] is adopted to account for the filtered reaction rate from the computation of the known instantaneous resolved flame front. The model parameter can be automatically adjusted based on the resolved flame front characteristics. For present investigation, the capability of the DFSD model is examined in predicting essential safety-related parameters and flame characteristics in a small-scale combustion chamber with repeated obstacles.

\section{Experimental Test Case}

The combustion chamber studied in this work was developed at the University of Sydney [5]. The schematic diagram of the laboratory-scale chamber is shown in Fig. 1 (left). The $50 \times 50 \times 250 \mathrm{~mm}$ combustion chamber can accommodate a maximum number of 3 baffle plates positioned at equidistance and a solid square obstacle of 12-mm cross section.
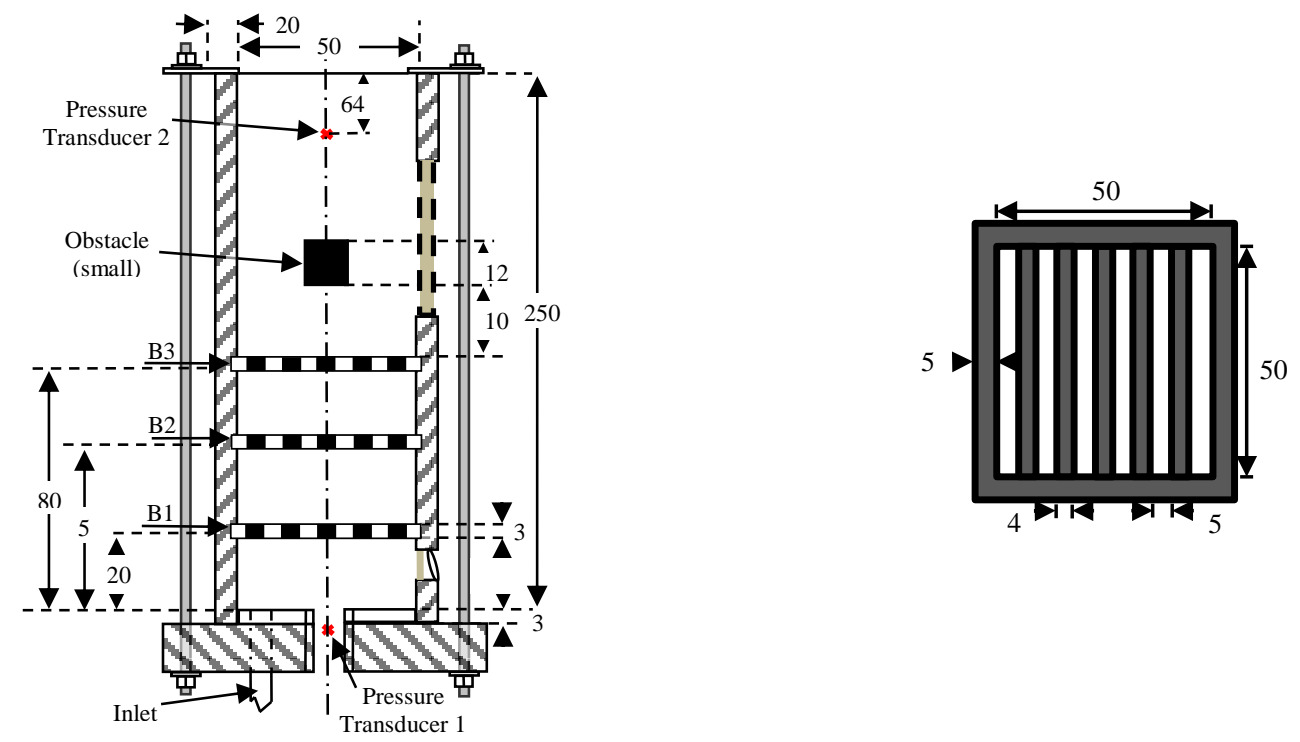

Fig. 1: Left: Schematic diagram of the Sydney combustion chamber [5]. Right: Removable baffle plates. Units are in millimetre. 
The schematic of the removable baffles is displayed in Fig. 1 (right). Each baffle plate is $3 \mathrm{~mm}$ thick, consisting of five $4 \mathrm{~mm}$ wide bars each with a 5-mm wide space spreading them throughout the chamber, creating a blockage ratio of 0.4. This chamber is of specific interest due to its smaller volume and its capability to hold a deflagrating flame, because of the strong turbulence environment generated by solid obstacles at different downstream locations from the closed bottom end. In the case considered here a LPG-air (treated as propane in LES) mixture is ignited by focusing an infrared output from a Nd:YAG laser $2 \mathrm{~mm}$ above the base. Venting at atmospheric pressure is maintained throughout the explosion process. For the present investigation, the configuration with all three baffles and solid obstacle is considered, as shown in Fig. 1 (left). The influence of the number and position of the obstructions is not discussed here but can be found elsewhere [6]. Experimental measurements recently published by Alharbi et al. [5] are used for validation of LES simulations.

\section{Mathematical Model}

LES calculations of propagating turbulent flames past repeated obstacles are performed using the in-house code PUFFIN [2]. Detailed description of numerical schemes and computational set-up is not presented here but can be found elsewhere [2,6]. Besides the conservation equations for mass, momentum and energy, the use of the dynamic flame surface model involves solving a transport equation for the filtered progress variable $\tilde{c}$ [4], as shown in Eq. (1)

$$
\frac{\partial \bar{\rho} \tilde{c}}{\partial t}+\frac{\partial}{\partial x_{i}}\left(\bar{\rho} \tilde{u}_{i} \tilde{c}\right)=\frac{\partial}{\partial x_{i}}\left(\frac{\rho_{u} \Xi_{\Delta} s_{L}^{0} \Delta}{16 \sqrt{6 / \pi}} \frac{\partial \tilde{c}}{\partial x_{i}}\right)+4 \rho_{u} s_{L}^{0} \sqrt{\frac{6}{\pi}} \Xi_{\Delta} \frac{\tilde{c}(1-\tilde{c})}{\Delta}
$$

where $\Delta$ is the filter width and $\Xi_{\Delta}$ is the sub-grid-scale wrinkling factor. The LES filter $\Delta$ should be larger than the mesh size $\Delta_{\mathrm{X}}$ (typically $\Delta \geq 5 \Delta_{\mathrm{X}}$ ) so that the flame can be sufficiently resolved. The diffusion term in Eq. (1) controls the filtered flame thickness. It is primarily designed to recover the correct flame propagation [4]. For present study, $\Delta=8 \Delta_{\mathrm{X}}$ is used. The reaction rate is expressed using the flame surface density approach and the SGS wrinkling factor $\Xi_{\Delta}$ is calculated as

$$
\Xi_{\Delta}=\left(\frac{\Delta}{\delta_{c}}\right)^{\beta}
$$

where the inner cut-off scale $\delta_{c}$ is prescribed by the user and should be of the order of the laminar flame thickness $\delta_{L}^{0}$ [7]. In the present work, $\delta_{c}=6 \delta_{L}^{0}$ is adopted. The model parameter $\beta$ should be obtained from the information of the resolved flame front. It is computed dynamically as [7]

$$
\beta \approx \frac{\ln (\langle|\widehat{\nabla \tilde{c}}|\rangle / \nabla \widehat{\widetilde{c}})}{\ln \left(\sqrt{1+(\widehat{\Delta} / \Delta)^{2}}\right)}
$$

Eq. (3) is derived from a Germano-like identity and makes use of the conservation of the total flame surface at filter and test filter scales [4]. Test-filtering procedure is realised by using a 3-D Gaussian filter at the test-filter size $\widehat{\Delta}=1.0 \Delta$. The volume averaging is also replaced by the Gaussian filter at the scale $\Delta_{\mathrm{m}}=1.5 \hat{\Delta}$ [7]. Note that the test-filter width should be at least of the size of the resolved flame front and averaging-filter width is typically larger than $\hat{\Delta}$. The dynamic flame surface density model has been implemented and tested in the PUFFIN code for the simulation performed in this paper. The input unstrained laminar burning velocity and flame thickness are set as $s_{L}^{0}=42.5 \mathrm{~cm} / \mathrm{s}$ and $\delta_{L}^{0}=0.37 \mathrm{~mm}$ [4], respectively, for the stoichiometric propane-air mixture. Turbulence is modelled using the dynamic Smagorinsky model [8].

Simulation is initiated by imposing a laminar flame kernel at the bottom centre of the combustion chamber. The initial $\tilde{c}$ profile across the flame front is given from the solution of steady laminar propagating flame for the LES filter $\Delta$. The radius of the initial kernel is set to $4.0 \mathrm{~mm}$. Three-dimensional LES was carried out using three numerical grids in order to examine the solution dependence on grid resolution. However, results for the fine computational mesh with 2.7 million 
grid points are presented in this paper. The computational domain is extended to $325 \mathrm{~mm}$ in $x, y$ and $250 \mathrm{~mm}$ in $z$ direction with the far-field boundary conditions. The average grid size within the chamber is about $0.7 \mathrm{~mm}$.

\section{Results and Discussion}

The LES results of stagnant, stoichiometric propane-air deflagrating flames over three baffle plates and a central square obstacle are presented and discussed in this section. It focuses on the ability of LES in predicting the pressure rise and flame acceleration, the flame characteristics in the deflagration and the influence of flame-obstacle interaction.

\subsection{Overpressure and Flame Propagation Speed}

Fig. 2 shows the overpressure history at the chamber base from LES and the experiment. Note that all the experimental pressure signals are plotted for validation. It can be seen that LES with the DFSD model is able to correctly predict the trend, rate of change and magnitude of overpressure, compared with experiments. The predicted time taken to reach the peak pressure (referred to as time-to-peak) is also within the range of experimental variations and is very close to the mean value. Furthermore, the predicted maximum overpressure is in good agreement with experiments. It is shown that the overpressure remains relatively low before $t=5 \mathrm{~ms}$. Thus, it may be considered as the early stage of flame propagation when the propagation of flame front is still slow. Subsequently, LES predicts a first obvious pressure rise, a significant increase and a pressure peak at around $\mathrm{t}=6,9$ and $10.5 \mathrm{~ms}$, respectively. Thereafter, the overpressure begins to drop, and it oscillates towards the atmospheric pressure due to acoustics. A similar trend of overpressure development can be also observed from the experiment.

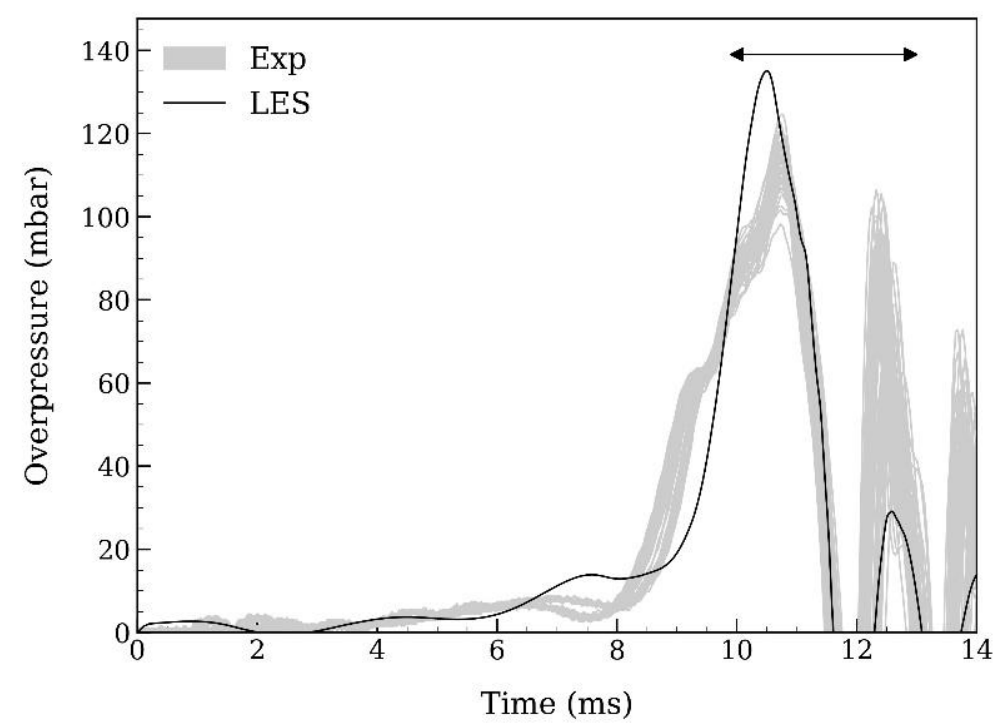

Fig. 2: Overpressure time history of LES and experiments. Arrow: variation of time taken to reach the peak pressure in experiments.

Fig. 3 shows the speed of flame leading point from LES and experimental measurements. Locations of the midpoints of the three baffle plates (B1, B2 and B3) and the central square obstacle (Sq.Ob.) are also indicated to identify the influence of the obstructions. In the experiment, the displacement of the flame front is derived from highspeed images. Speed of the leading point is then computed knowing that the high-speed imaging system has a repetition rate of $5 \mathrm{kHz}$. Numerically, the speed of flame leading edge is calculated from the extracted flame positions using the same time interval of $0.2 \mathrm{~ms}$. Throughout the deflagration, the flame-front speed increases significantly from below $5 \mathrm{~m} / \mathrm{s}$ close to the bottom end of the chamber to above $150 \mathrm{~m} / \mathrm{s}$ near the top exit, indicating the strong acceleration caused by the blockage of obstruction. It should be noticed that the flame is initially laminar and expands hemi-spherically before touching the side walls of the chamber. Flame propagation at this stage is dominated by the 
laminar burning velocity $s_{L}$ and the thermal expansion factor $\Theta$. Note that both of them are intrinsic thermo-chemical properties of the unburned mixture instead of turbulence. Evidently from both experiment and LES, the flame speed increases when it approaches an obstacle. However, the flame tends to decelerate between obstructions despite of the relatively high congestion. The level of flame acceleration is closely related to the turbulence intensity at various locations of the chamber. It can also be observed that the flame accelerates dramatically around the third baffle due to high turbulence level, leading to strong flame-turbulence interaction. At this stage, the flame jets out of the third baffle and encounters the square obstacle. After passing the central square obstacle, the flame speed starts to increase in a quasisteady manner towards the chamber exit. Evidently, LES with the DFSD model excellently reproduces the essential dynamic behaviours of the flame in the deflagration.

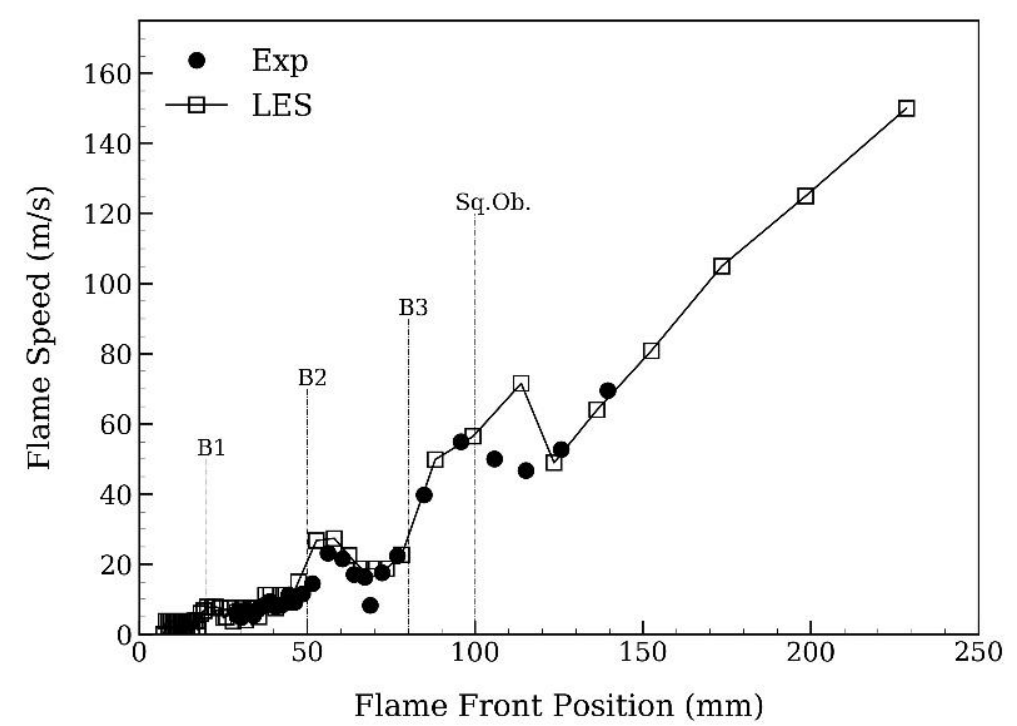

Fig 3: Flame propagation speed of LES and experiment. B1, B2 and B3: first, second and third baffle plates. Sq.Ob.: Square Obstacle.

\subsection{Role of the SGS Wrinkling Factor}

A major advantage of the DFSD model is the capability of quantifying the flame-turbulence interaction at the sub-grid scale using the SGS wrinkling factor $\Xi_{\Delta}$. Note that in LES, the large-scale vortices and turbulence are resolved on the grid and their effect on the flame stretching is directly taken into account. SGS wrinkling factor essentially quantifies the effect from the SGS vortices or turbulence with respect to the resolved contribution. Fig. 4 shows the evolution of the flame wrinkling factor as a function of flame front position. The 'local' wrinkling factor is extracted from the leading point of the flame (the maximum distance from the ignition end where $\tilde{c}=0.5$ is present), while the 'averaged' $\Xi_{\Delta}$ is computed by performing volume-averaging within the flame (defined as $0.05<\tilde{c}<0.95$ here). Initially, both 'local' and 'averaged' wrinkling factors are close to unity as the flame is essentially laminar. Fluctuation of local flame-front wrinkling can be observed as the flame continuously propagates past the three baffles and the square obstacle. The trend and magnitude were found to be dependent on the parameters of the test-filtering procedure including the size of the test filter $\widehat{\Delta}$ and the inner cut-off scale $\delta_{c}$.

Note that combustion of the fresh gases induces flow and turbulence typically in the wake area of the obstruction ahead of the flame. The propagating flame front then interacts with the vortices and turbulent eddies, leading to distortion and wrinkling. This increases the flame area and the burning rate. Consequently, the fast displacement of the unburned gases generates higher turbulence in the presence of confinement and obstruction. It in turn promotes the combustion rate. The process can be identified from the evolution of the wrinkling factor in the flame propagation.

Although $\Xi_{\Delta}$ of the flame leading point fluctuates significantly between obstacles, the level of flame wrinkling varies over the flame. The flame-averaged $\Xi_{\Delta}$ represents the total contribution from the SGS turbulence effects on the entire 
flame. Fig. 4 confirms that the averaged $\Xi_{\Delta}$ progressively grows from unity and its rate of change depends on the flow conditions ahead of the flame. In general, increase in propagation speed due to the local turbulence causes further stretching and wrinkling of the flame front. It can also be observed that the averaged wrinkling factor tends to stabilise around $\Xi_{\Delta} \approx 1.8$. This means that the SGS contribution of flame-turbulence interaction to the burning rate has reached equilibrium. It may also explain the nearly constant flame acceleration towards the chamber exit, as can be noticed from Fig. 3.

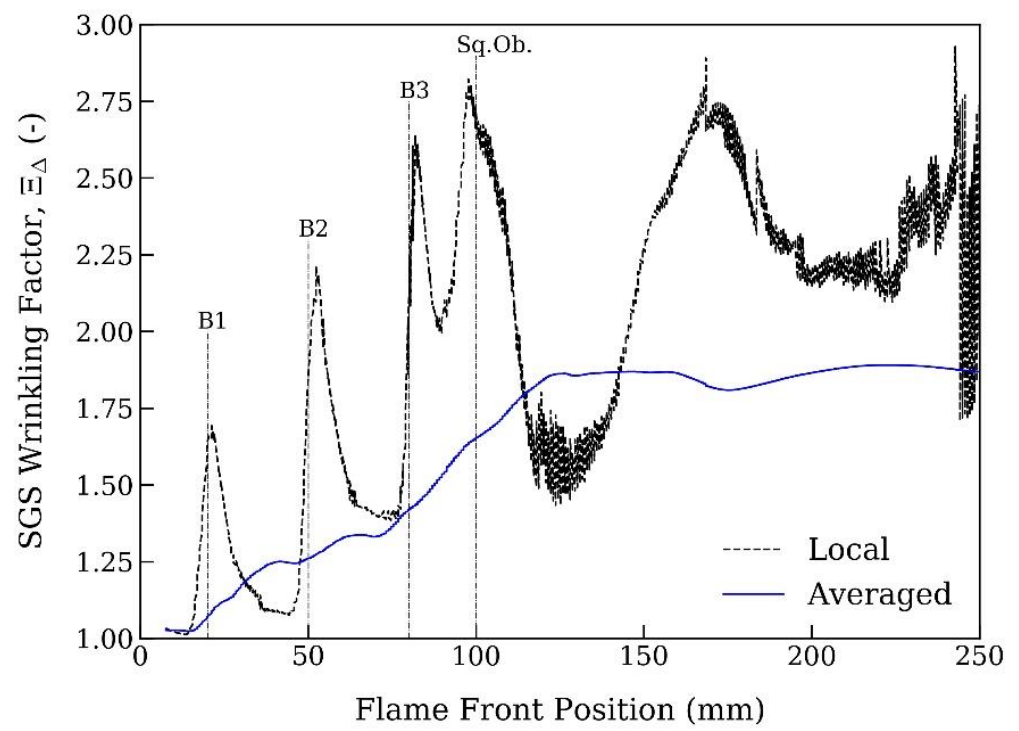

Fig. 4: Evolution of flame wrinkling factor $\Xi_{\Delta}$ from LES. Local: value at the flame leading point. Averaged: volumeaveraged value across the flame.

Fig. 5 contains two three-dimensional flame snapshots of the early $(t=5.1 \mathrm{~ms})$ and later $(\mathrm{t}=10.1 \mathrm{~ms})$ stages of deflagration when the flame is passing the first and the last obstacles, respectively. The flame is contoured by the SGS flame wrinkling factor $\Xi_{\Delta}$. The level of flame wrinkling may indicate the strength of local interaction between the flame front and the surrounding turbulent eddies. As can be seen from the flame at its early stage, it almost remains quasi-laminar after passing through the first baffle with $\Xi_{\Delta}$ close to unity over most of the flame region. The corresponding overpressure is also relatively low. This is mainly due to the relatively weak turbulence behind the first baffle plate. The flame is then wrinkled by the vortices around the obstacles downstream and it can be noticed that $\Xi_{\Delta}$ is generally much higher in the fully turbulent phase, e.g. at $\mathrm{t}=10.1 \mathrm{~ms}$. In the later phase of flame propagation, the region of strong flame-turbulence interaction has $\Xi_{\Delta} \geq 2$ and is mainly distributed behind the third baffle. On the contrary, the part of the flame between the ignition end and the second baffle remains quasi-laminar with $\Xi_{\Delta} \approx 1$. Simple calculation shows that with $\Xi_{\Delta} \geq 2.5$, more than $60 \%$ of the local reaction rate is from the sub-grid scale. Hence, the high turbulence from the third baffle downstream leads to high peak overpressure and fast flame propagation. The spatial variation of the SGS wrinkling factor confirms the regions of weak and strong flame-flow interactions in the combustion chamber. It reveals the significance of appropriately accounting for the SGS part of the filtered reaction rate in LES so that the flame characteristics can be sufficiently captured in the fully turbulent phase and the behaviours of laminar flame at the early stage can also be recovered. 


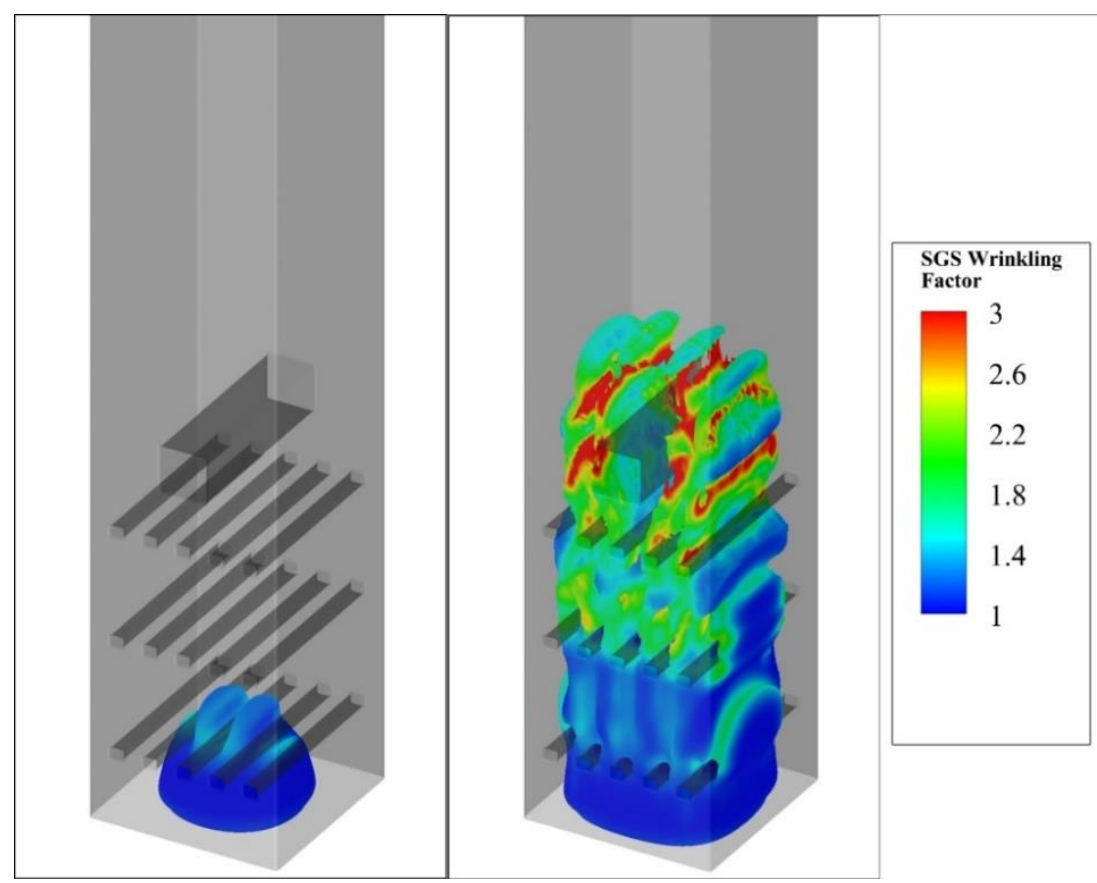

Fig. 5: Snapshots of flame (iso-surface of $\tilde{c}=0.5$ ) from LES contoured by the wrinkling factor at $\mathrm{t}=5.1$ and $10.1 \mathrm{ms,}$ respectively.

\section{Conclusion}

A LES-based numerical study was carried out to study the pressure rise and flame acceleration in vented deflagration with repeated obstacles. The experimental test case is a small-scale combustion chamber with three baffle plates and a central square obstacle. For present investigation, a stagnant, stoichiometric propane-air mixture was considered. In LES, the filtered reaction rate was modelled using a novel dynamic flame surface density model. The performance of the model was assessed by comparing the results of overpressure history and flame-front speed against the published experimental data. It was confirmed that LES with the DFSD model is capable of reproducing the essential safety-related parameters including the trend and magnitude of the overpressure. LES was also successful in predicting characteristics of the flame propagation at different stages of the deflagration such as the flame speed. Strength of SGS flame-turbulence interaction was identified using the sub-grid-scale wrinkling factor and it evolves significantly from the early quasi-laminar to the later fully turbulent phase. The strong flame stretch caused by the obstacle-generated vortices and turbulence was found to result in large acceleration of the flame front. It also contributes to the substantial rise of the internal pressure of the combustion chamber. The effect of small-scale vortices on the burning rate and flame speed has been found to be significant in the regions of high turbulence intensity such as the wake area of the square obstacle. The study also highlights the importance of the SGS combustion model in simulating turbulent premixed propagating flames past obstructions.

\section{References}

[1] S. R. Gubba, S. S. Ibrahim, W. Malalasekera, and A. R. Masri, "LES Modeling of Premixed Deflagrating Flames in a Small-Scale Vented Explosion Chamber with a Series of Solid Obstructions," Comb. Sci. and Tech., vol. 180, no. 10-11, pp. 1936-1955, 2008.

[2] M. P. Kirkpatrick, S. W. Armfield, A. R. Masri, and S. S. Ibrahim, "Large Eddy Simulation of a Propagating Turbulent Premixed Flame," Flow, Turbul. Combust., vol. 70, pp. 1-19, 2003.

[3] M. Boger, D. Veynante, H. Boughanem, and A. Trouvé, "Direct numerical simulation analysis of flame surface density concept for large eddy simulation of turbulent premixed combustion," Symp. Combust., vol. 27, no. 1, pp. 917-925, 1998.

[4] G. Wang, M. Boileau, D. Veynante, and K. Truffin, "Large eddy simulation of a growing turbulent premixed flame kernel using a dynamic flame surface density model," Comb. Flame, vol. 159, no. 8, pp. 2742-2754, 2012.

[5] A. Alharbi, A. R. Masri, and S. S. Ibrahim, "Turbulent premixed flames of CNG, LPG, and $\mathrm{H}_{2}$ propagating past 
repeated obstacles," Exp. Therm. Fluid Sci., vol. 56, pp. 2-8, 2014.

[6] S. R. Gubba, S. S. Ibrahim, W. Malalasekera, and A. R. Masri, "Measurements and LES calculations of turbulent premixed flame propagation past repeated obstacles," Comb. Flame, vol. 158, no. 12, pp. 2465-2481, 2011.

[7] D. Veynante and V. Moureau, "Analysis of dynamic models for large eddy simulations of turbulent premixed combustion," Comb. Flame, vol. 162, no. 12, pp. 4622-4642, 2015.

[8] M. Germano, "Turbulence : the filtering approach," J. Fluid Mech., vol. 238, 1992. 